\title{
The Imaging X-ray Polarimetry Explorer (IXPE)
}

\author{
Riccardo Ferrazzoli*i \\ INAF-IAPS \\ E-mail: riccardo.ferrazzoli@inaf.it
}

\begin{abstract}
X-ray polarimetry is a long-standing missing piece in the puzzle of multiwavelenght study of high energy sources. The Imaging X-ray Polarimetry Explorer (IXPE) will fill this gap in our knowedge by adding polarization measurements to observables currently avaiable: energy, time, and position. IXPE, first proposed in response of an Announcement of Opportunity issued on 2014, was selected in the context of the NASA Astrophysics Small Explorer (SMEX) program in January 2017, and is a collaboration with the Italian Space Agency (ASI). Thanks to its Gas Pixel Detector imaging-capable polarimeter, IXPE will open new a new window in the understanding of particle acceleration processes, higly magnetized compact objects and the properties of scattering induced polarization. Here, the thecnical aspects of the mission and some of the scientific cases that IXPE will explore will be highlighted. Special attenton will be given to a selection of extended sources that IXPE will be able to study thanks its imaging capabilities.
\end{abstract}

Multifrequency Behaviour of High Energy Cosmic Sources - XIII - MULTIF2019

3-8 June 2019

Palermo, Italy

\footnotetext{
${ }^{*}$ Speaker.

${ }^{\dagger}$ On behalf of the IXPE collaboration.
} 


\section{X-ray Polarimetry: the missing piece of the puzzle}

Polarimetry, from radio to optical, has always been an invaluable tool for the understanding of the geometry of High Energy cosmic sources and the physical processes powering them.

Taking for example a multifrequency look at the Crab Nebula (Fig. 1), polarization maps have been obtained in the radio, infrared and optical, allowing to describe and map the magnetic field properties on large scales.

On the other hand, when we come to the X-rays, we lack information on polarization for all but one source, i.e. the Crab Nebula itself. The sole positive detection of non-solar X-ray polarization known so far, is in fact due to the OSO-8 mission [19] [20]: this satellite-based Bragg X-ray polarimeter measured in the ' 70 s a linear polarization degree $P=19.2 \pm 1.0 \%$ and a polarization position angle $\theta=156.4 \pm 1.4^{\circ}$ at $2.6 \mathrm{keV}$. This result, while important since it demonstrated the synchrotron origin of the nebula's X-ray emission, is integrated over the whole nebula and lacks the depth and level of detail that polarimetry can reach in other wavelengths.

The fact that the polarization direction is not aligned to the jet suggests that its the result of the combination of many components such as the jet, tours, disk and the pulsar.

Since then, no other X-ray polarimetry missions has flown, and our knowledge of source geometry and emission mechanisms on the small scales from which the X-ray photons are emitted has remained incomplete.

Finally, at the beginning of the 2000's, the advent of photoelectric polarimeters [6] [3] brought a renown interest in X-ray Polarimetry. Compact, imaging-capable detectors with two order of magnitude increase of sensitivity with respect to the OSO-8 polarimeters became available and new missions are being designed around them.

The first mission entirely dedicated to X-ray polarimetry will be the Imaging X-ray Polarimetry Explorer (IXPE, [21]).

\section{The Imaging X-ray Polarimetry Explorer (IXPE)}

IXPE is born from a collaboration between the NASA Marshall Space Flight Center (MSFC) and the Italian Space Agency (ASI), it will improve the sensitivity of the OSO-8 polarimeter by two orders of magnitude and adding at the same time imaging capabilities, filling the gap with the polarimetric information available in other wavelengths.

It was selected in January 2017 as an Astrophysics Small Explorer (SMEX) mission after being successfully proposed in 2014 and is slated to be launched in April 2021.

NASA holds the PI-ship in the person of Martin Weisskopf and manages the X-ray mirrors design and fabrication, the Science Operation Center and telescope calibrations. Scientific data distribution and archiving will be managed by the NASA High-Energy Astrophysics Science Archive Research Center (HEASARC).

The Laboratory for Astronomy \& Space Physics of Boulder will handle the Mission Operations. Italian responsibilities are divided between ASI, INAF-IAPS, INFN and OHB-I and include the instrument management, Detector Units and Detector Service Units manufacturing and calibration. Italy also provides the track reconstruction algorithm and the Malindi Ground Station as the primary contact point for command, telemetry and data downlink. 

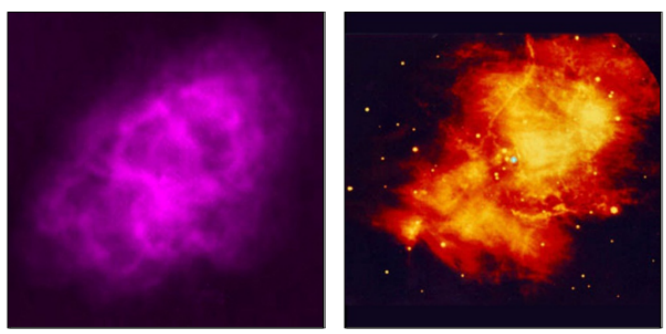

Radio (VLA)

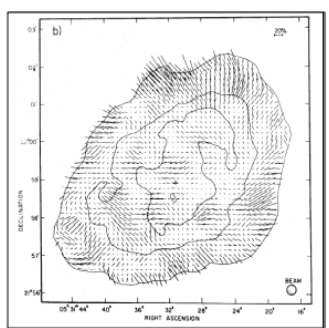

Radio polarization
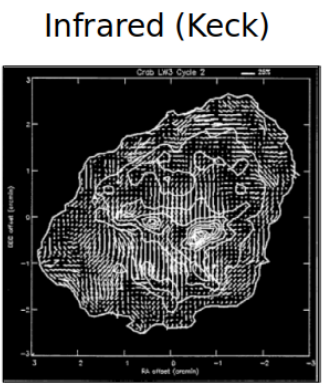

IR polarization

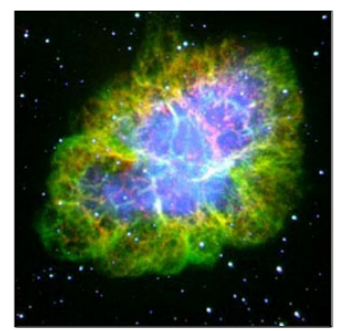

Optical (Palomar)

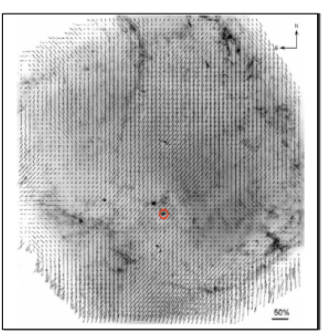

Optical polarization

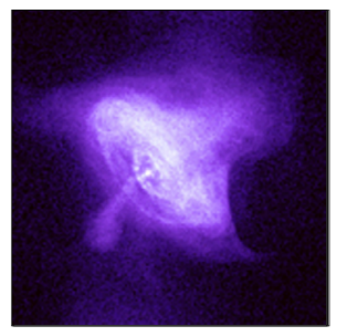

X-rays (Chandra)

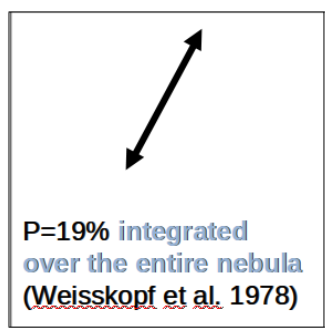

X-ray polarization

Figure 1: Multifrequency view of the Crab Nebula (Top row) and relative polarization maps (Bottom row) [7].

Ball aerospace will build the spacecraft.

A network of international scientific collaborators, institutions and Universities (such as Università Roma Tre, Stanford University, McGill University, and the Massachusetts Institute of Technology) provides the backbone of the Scientific Topical Working Groups, tasked with the selection of the sources to be put in the IXPE observing program, as well as the development of the theoretical predictions and analysis and interpretation of the data obtained during the observations.

The baseline IXPE mission calls for two years of science operations that can be further extended pending NASA approval.

It will be launched with a SpaceX Falcon9 rocket and placed in a $540 \mathrm{~km}$ circular, near-equatorial orbit in order to minimize the passage over the South Atlantic Magnetic Anomaly and maximize the number of passages per day over the Malindi Ground Station.

The scientific payload consist in three identical grazing-incidence X-ray telescopes with a $4 \mathrm{~m}$ focal length co-aligned to forward star trackers. Each telescope is made from 24 nested monolithic two-reflection electroformed shells of nickel-cobalt alloy (see Fig. 3(a)). The total effective area is $>700 \mathrm{~cm}^{2}$ at $3 \mathrm{keV}$, approximately flat up to $7 \mathrm{keV}$ (see Fig. 3(b)). At the focus of each telescope there is a Detector Unit containing a Gas Pixel Detector. The polarization sensitivity is such to reach a Minimum Detectable Polarization at $99 \%$ confidence $<5.5 \%$ for a $0.5 \mathrm{mCrab}$ source with a 10 day observation. The spurious modulation for an unpolarized source is expected to be $<0.3 \%$. The Angular resolution is $\sim 20^{\prime \prime}$ over a $10^{\prime}$ overlapping field of view for the three detectors' polarization sensitive areas. The energy band is the "classical" X-ray one of $2-8 \mathrm{keV}$ with an energy resolution of $\sim 20 \%$ at $5.9 \mathrm{keV}$. A timing accuracy of $20 \mu$ s can be reached using GPS-pulse-persecond signal and on-board clocks.

A summary of IXPE performance and characteristics is reported in Table 2. 


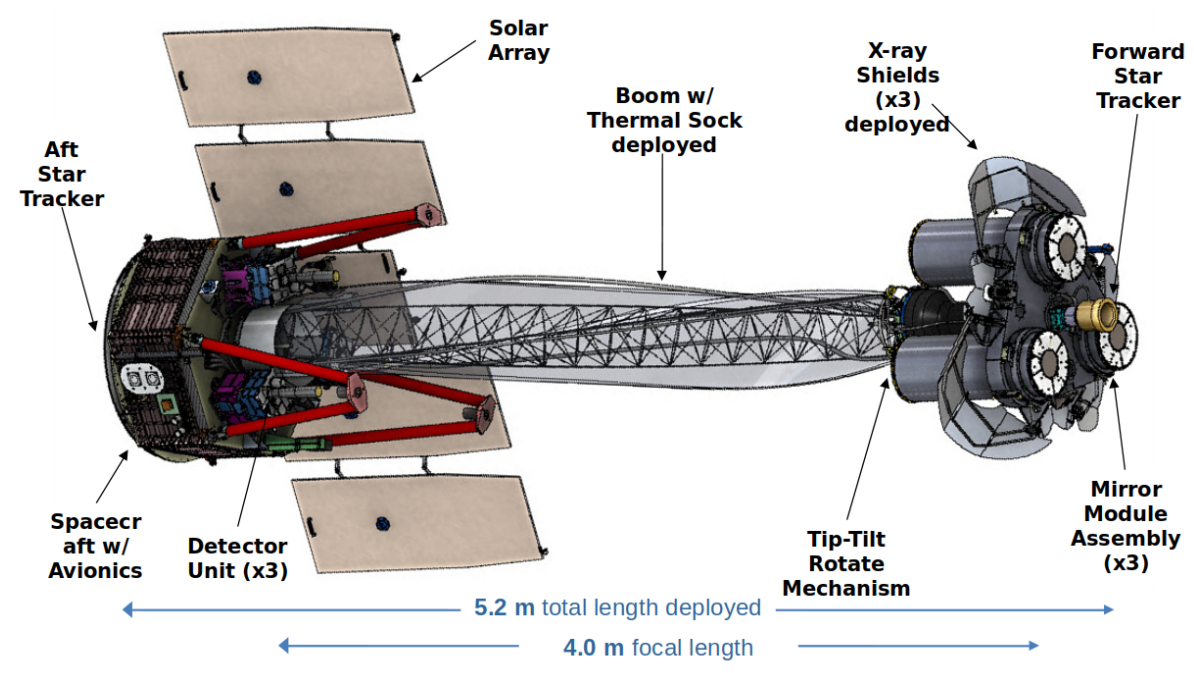

Figure 2: IXPE in deployed configuration.

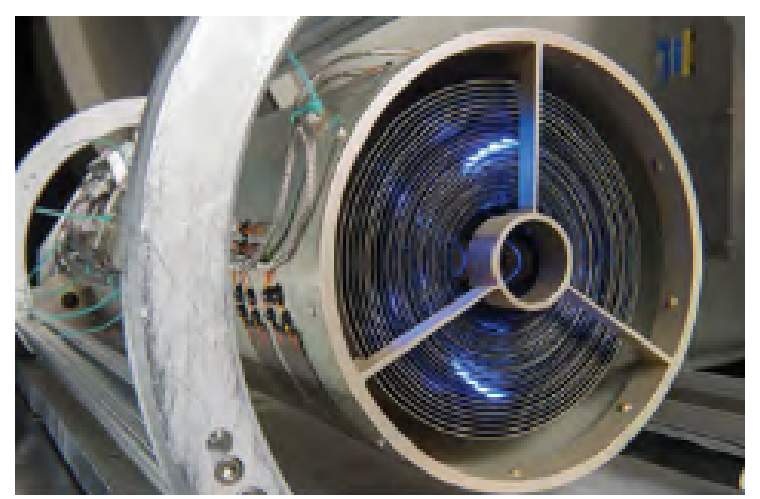

(a)

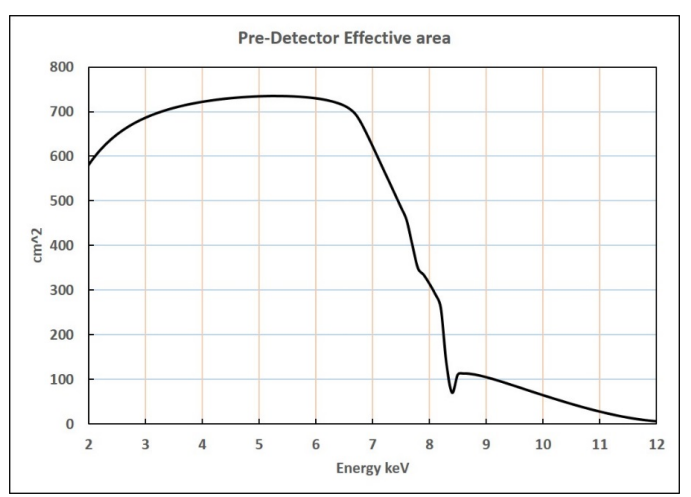

(b)

Figure 3: (a) Prototype of an IXPE Mirror Module Assembly [21]. (b) Effective area of the IXPE optics as a function of the energy.

\begin{tabular}{c|c} 
Parameter & Performance \\
\hline Launch date & April 2021 \\
Operational phase & 2 years +1 \\
Orbit & Near-equatorial, circular at $540 \mathrm{~km}$ \\
Polarization sensitivity & $M D P_{99}<5.5 \%$, for 0.5 mCrab in 10 days \\
Spurious modulation & $<0.3 \%$ \\
Effective area & $\sim 700 \mathrm{~cm}^{2}$ at $5.9 \mathrm{keV}$ \\
Angular resolution & $\sim 20^{\prime \prime}$ \\
Field of view & $\sim 10^{\prime}$ \\
Energy band & $2-8 \mathrm{keV}$ \\
Resolution & $\sim 20 \%$ at $5.9 \mathrm{keV}$ \\
Timing accuracy & $20 \mu \mathrm{s}$ \\
\hline
\end{tabular}

Table 1: Summary of IXPE performance and characteristics. 


\subsection{The Gas Pixel Detector}

The heart of the mission is the Gas Pixel Detector (GPD) imaging X-ray polarimeter [6] [3]. The GPD is based on the photoelectric effect: when an X-ray photon is absorbed in the drift region, a photoelectron is ejected, losing energy collisionally ionizing the gas in the cell, and producing an ionization track of electrons. This ionization track drifts to the Gas Electron Multiplier (GEM), where a strong electric field multiplies the number of electrons by a large factor. The amplified electron ionization track then transfers onto the pixelated anode, which is a $300 \times 352$ array of hexagonal pixels at $50 \mu \mathrm{m}$ pitch. The drift region is $1 \mathrm{~cm}$ thick and filled with dimethyl ether (DME) gas which has a small diffusion coefficient that helps maintain the spatial information on the track during the drift of the electrons to the GEM. A $50 \mu \mathrm{m}$ thick beryllium window lets the $\mathrm{X}$-ray enter the detector. A scheme of the GPD is shown in Fig. 4(a).

Since the photo-electron is emitted preferentially in the plane perpendicular to the photon polarization vector, by measuring the photoelectron emission direction one can reconstruct the polarization vector of the incident photon. Fig. 4(b) shows an actual GPD ionization track: processing algorithms applied to the track extract the location on the detector where the photon was absorbed, its energy (proportional to the total charge in the track), and the initial direction of the photoelectron. With this technique all four characteristics of the incident radiation, i.e. polarization, incoming direction, energy, and time, can be measured at the same time.

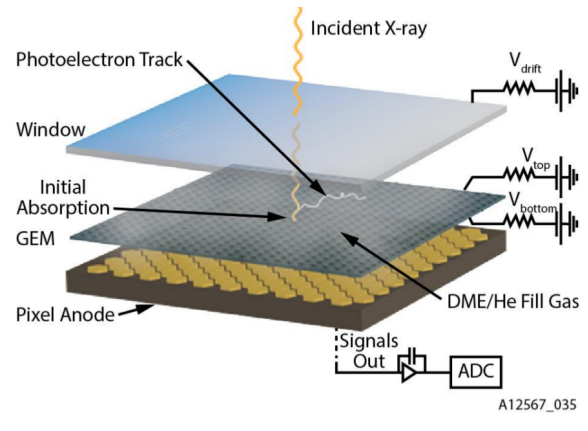

(a)

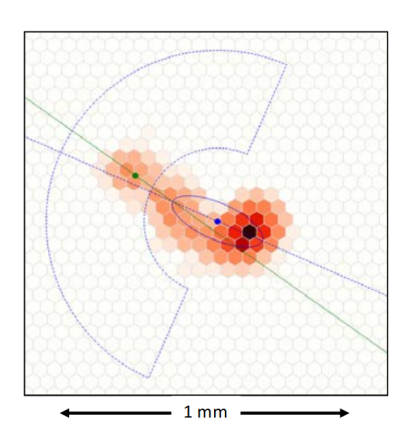

(b)

Figure 4: (a) Exploded view of a Gas Pixel Detector [9]. (b) Photoelectron track in a GPD.

\subsection{In-Flight calibration system}

The GPD is a technologically mature, having been tested for more than 15 years, but complex instrument, so the study of its behavior during the mission lifetime will be a crucial task. For this reason each Detector Unit (DU) includes a Filter and Calibration Wheel (FCW, [13]), which hosts the Filter and Calibration Set (FCS). The FCS includes four polarized and unpolarized calibration sources to monitor GPD performance in flight and filters for special observations. All calibration sources inside the FCW contains a $\mathrm{Fe}^{55}$ nuclide, whose activity naturally decays with half time of 2.7 years. The DU calibrations will be performed in orbit during the Earth occultation of the celestial sources in order to: (1) map and monitor the modulation factor value of the GPD for monochromatic photons, and hence the stability of polarimetric response, at two energies $(3.0 \mathrm{keV}$ 
and $5.9 \mathrm{keV})$; (2) map and monitor the energy resolution of the GPD; (3) check for the presence of spurious polarization; (4) map and monitor the gain of the Gas Electron Multiplier and its nonhomogeneities. The four calibration sources which can be alternatively put in front of the GPD, shown in Fig. 5(a), are:

- Calibration source A (CalA): This source produces polarized X-ray photons at two energies in the IXPE band with known polarization state in order to monitor the modulation factor of the instrument. The source is shown in Fig. 5(b). A Fe ${ }^{55}$ nuclide mounted into a T-shaped holder produces X-rays at $5.9 \mathrm{keV}$ and $6.5 \mathrm{keV}$ that partially absorbed by a $1.6 \mu \mathrm{m}$ thick silver foil in order to produce fluorescence at $2.99 \mathrm{keV}$ and $3.15 \mathrm{keV}$. Photons at $3.0 \mathrm{keV}$ and $5.9 \mathrm{keV}$, collimated with a broad collimator, are Bragg diffracted on a graphite mosaic crystal, with FWHM mosaicity of $1.2 \mathrm{deg}$, at first and second order of diffraction, approximately at the same diffraction angle ( $38.3 \mathrm{deg}$ and $38.7 \mathrm{deg}$ respectively) and hence polarization. A second collimator is used to block stray-light X-rays.

- Calibration source B (CalB): This source produces a collimated beam of unpolarized photons at $5.9 \mathrm{keV}$ to monitor the absence of a spurious modulation. A diaphragm with an aperture of $1 \mathrm{~mm}$ collimates X-rays to produce on the GPD a spot of about $3 \mathrm{~mm}$ which is representative of the image of a point-like source when the spacecraft pointing dithering is included.

- Calibration source $\mathbf{C}$ (CalC): This source illuminates all the detector sensitive area to map the gain at one energy: $5.9 \mathrm{keV}$.

- Calibration source D (CalD): This source illuminates all the detector sensitive area as CalC, to map the gain at a different energy. The $\mathrm{Fe}^{55}$ source illuminates a silicon target to extract its $K_{\alpha}$ and $K_{\beta}$ fluorescence at $1.7 \mathrm{keV}$, which then impinges on the detector. Since photoelectron tracks are smaller at this energy, gain maps can achieve a higher spatial resolution. The design is such that X-ray photons from $\mathrm{Fe}^{55}$ can not directly impinge on the GPD sensitive area to avoid detector saturation.

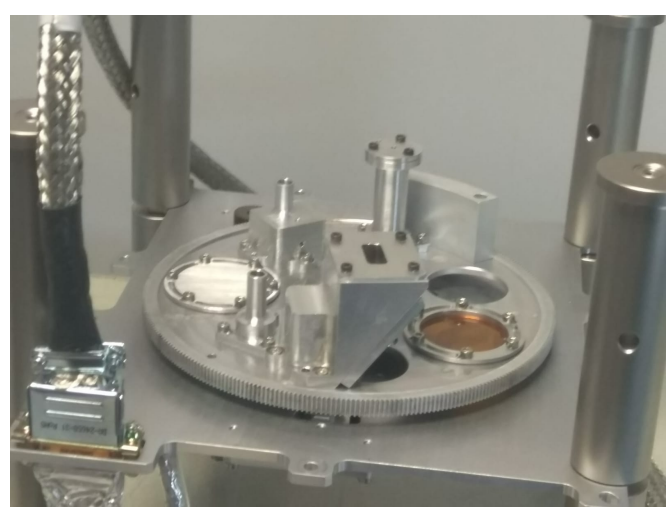

(a)

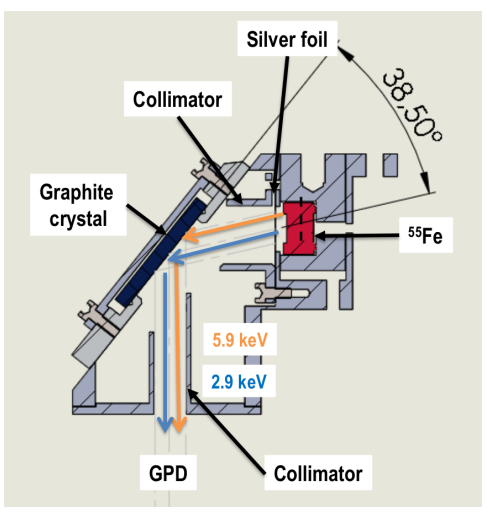

(b)

Figure 5: (a) View of the Filter and Calibration wheel: from the left, clockwise, the closed position, CalD, CalB, the Open position, the Gray Filter, CalA, CalC. (b) Cut image of CalA. 


\section{Science with IXPE}

With the birth of observational X-ray astronomy in the early 60's many sources were found to be characterized by non-thermal emission processes and/or by radiation transferred in highly asymmetric systems. It is then expected that almost every X-ray source will exhibit polarization properties.

The science cases available to IXPE can be divided in the following categories:

- Particle acceleration processes (Pulsar Wind Nebulae, Supernova Remnants, Jets and Blazars, Microquasars...);

- Highly magnetized compact objects (Rotation powered and accreting pulsars, magnetars...);

- Scattering induced polarization (X-ray binaries, Radio Quiet Active Galactic Nuclei, Molecular Clouds in the Galactic Center...);

- Fundamental physics (Quantum Electro Dynamics, Strong \& Quantum gravity, Axion-Like Particles...).

The observation program calls for dithered observations of dozens of targets that will last from less than a day for the brightest sources to weeks for the faintest.

All the scientific data will be publicly available one week after the observation, after data validation, so there will be no proprietary period. Target of opportunity observations will be possible with a few-days response. The list of sources to be observed and the duration of their observations it's being finalized by the scientific topical working groups.

In the next sections, a showcase of some of the scientific cases available to IXPE relative to the study of extended sources will be presented.

\subsection{Cosmic Rays acceleration sites in Supernova remnants}

Supernova Remnants (SNR) are a prime target to study regions of particle acceleration trough polarimetric imaging. Young SNR are infact relativistic particle accelerators and the most likely sources of Galactic cosmic rays [15]. The acceleration mechanism is generally accepted to be diffusive shock acceleration (DSA, [10]), in which charged particles are repeatedly reflected by magnetic mirrors.

In several SNRs X-ray synchrotron emitting regions where electrons are accelerated up to $10-100$ $\mathrm{TeV}$ have been identified [4]. At such energies, the time scale of radiation loss is very short so that $\mathrm{X}$-ray synchrotron emission can only occur in regions of active particle acceleration, such as the SNR shock fronts, where magnetic fields are considerably strong and turbulent. In fact according to DSA theory the magnetic-field turbulence plays a crucial role, being created by the cosmic rays themselves as they diffuse ahead of the shock front.

The X-ray synchrotron emitting regions can be very narrow (up to a few arcseconds), with their widths being a measure of the average strength of the magnetic field: typical values range between $100-500 \mu \mathrm{G}$, well above the Galactic field strength of $\sim 5 \mu \mathrm{G}$, indicating strong magnetic field amplification [2]. 
Spatially resolved X-ray polarimetry could separate the thermalized plasma from non-thermal (synchrotron) components, locate the regions of ordered magnetic field on the site of shock acceleration and thus explore the turbulence level of the magnetic field: if polarized X-ray emission were to be detected from a SNR, it would provide unique constraints on models of diffusive shock acceleration with efficient magnetic field amplification.

One of the most interesting targets that can be studied to this end is the Tycho SNR, also known as SN 1572. Tycho is a young SNR that exhibits peculiar structures in the $4-6 \mathrm{keV}$ band, such as the so called "stripes" in the eastern rim, thought to be cosmic rays acceleration sites (see Fig. 6(a)). In Fig. 6 is shown a simulated IXPE observation of the Tycho SNR using a toy input model based on the Chandra $4-6 \mathrm{keV}$ image and input maps of polarization fraction and angle, and assuming an observation time of $500 \mathrm{ks}$, highlighting the prominence of the non-thermal polarized regions in the eastern rim of the remnant.

Tycho, together with CasA and SN1066 will be part of the IXPE first year observation plan.

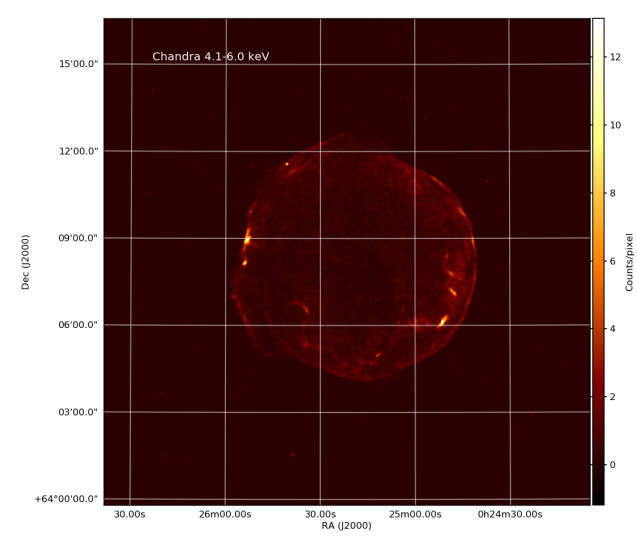

(a)

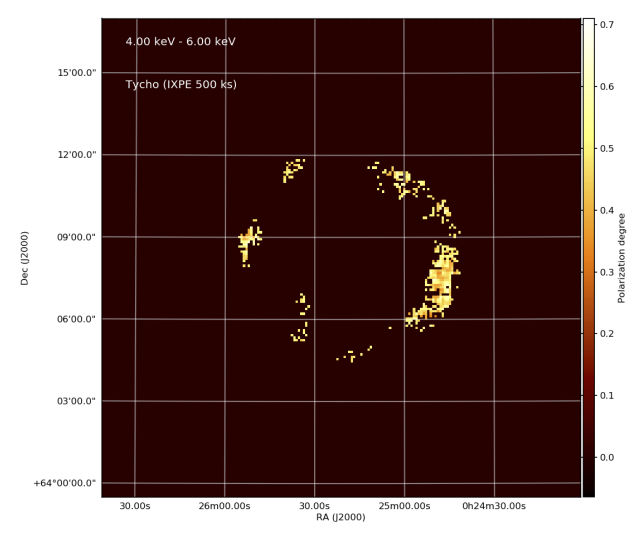

(c)

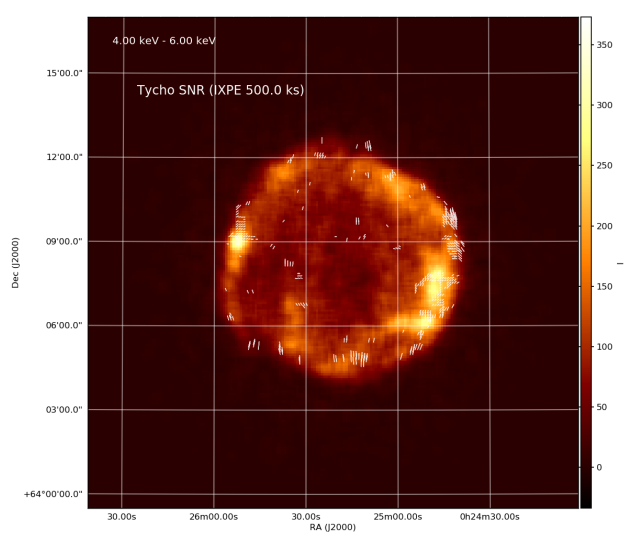

(b)

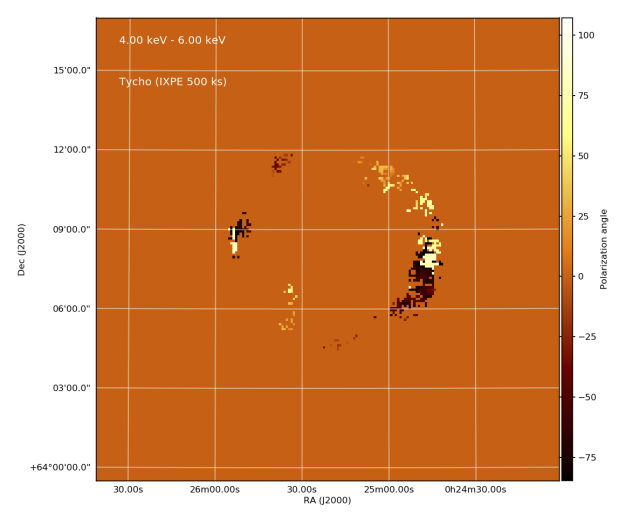

(d)

Figure 6: (a) Tycho SNR in the 4-6 kev band as seen by Chandra. (b) IXPE simulated observation of Tycho in the 4-6 kev band with superimposed polarization arrows. (c) Polarization Degree map of Tycho SNR. (d) Polarization Angle map of Tycho SNR. 


\subsection{Acceleration mechanisms in Jets: Centaurus A}

Jets are collimated outflows of highly relativistic plasma which are commonly observed in Active Galactic Nuclei and a number of X-ray binaries. The closest and brightest radio-loud AGN have jets that can be directly imaged in X-rays on arcmin scales (M87 and Centaurus A in particular, see Fig. 7(a)). The jets are characterized by bright spots, known as knots: the knots are thought to be the sites of enhanced emission where shocks are likely responsible for the acceleration of the emitting particles. Their emission from radio to optical is well described by synchrotron radiation, which explains their non-thermal spectra and the high degree of radio and optical polarization which is often measured. The emission mechanism in the X-ray energies is however still not constrained and X-ray polarimetry will be crucial, as the expected polarization signature strongly depends on the emission model. If it is synchrotron, the baseline model is that the polarization should be similar to the radio-optical, provided the emitting regions at the different ranges are cospatial. For X-rays produced by Synchrotron Self Compton (SSC) the incoming photon field is polarized giving rise to polarized $\mathrm{X}$-rays with polarization degree depending on the seed photon distribution and inclination. In the case of External Compton, the seed photons may come from the disk and/or the CMB and yield polarization signatures of the order of a few \% [12]. In Fig. 7(b) is shown a simulated 2 Ms IXPE observation of Centaurus A, demonstrating the possibility of resolving the Jet from the Core and perform X-ray polarimetry along the Jet.

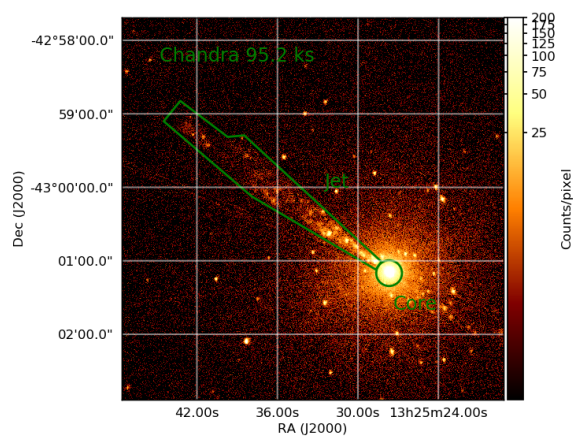

(a)

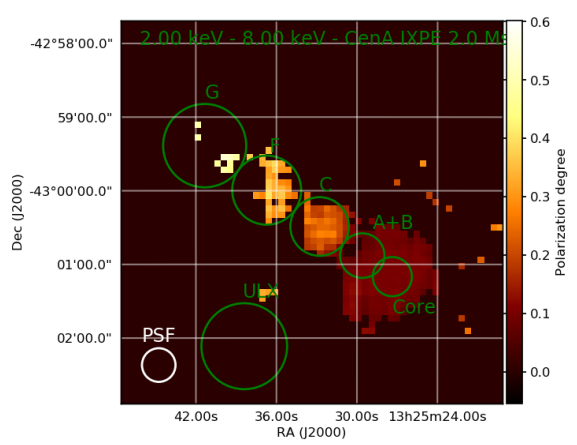

(c)

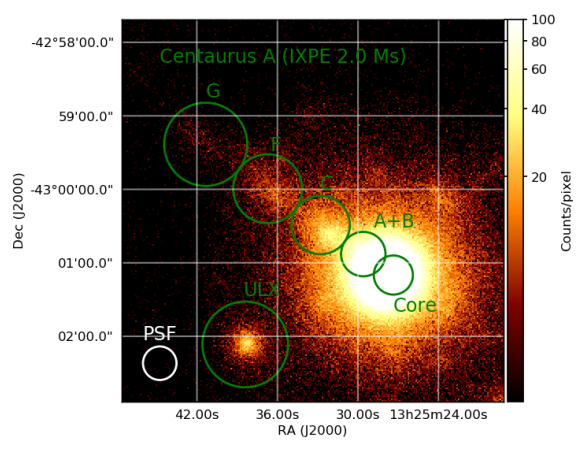

(b)

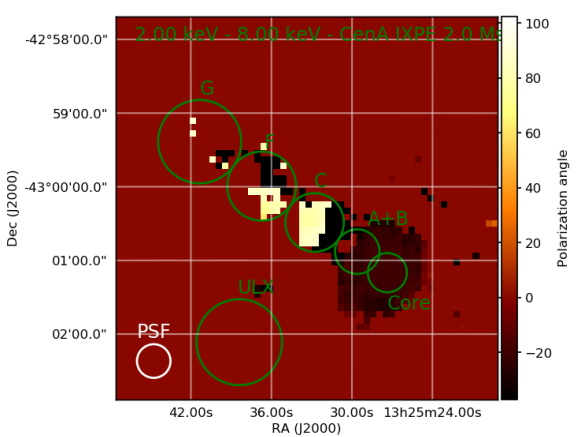

(d)

Figure 7: Simulation of a 2 Ms IXPE observation of Centaurus A (b) compared with a Chandra observation of the same source (a). (c) Polarization Degree map of Centaurus A. (d) Polarization Angle map of Centaurus A. 


\subsection{Galactic Archaeology}

One of the most fascinating possibilities that IXPE opens up, is the one of performing a sort of Galactic Archaeology.

SgrA*, the supermassive black hole in the center of our Milky Way is remarkably quiescent and Xray dim, with an emission that is just $10^{-11}$ of its Eddington luminosity[1]. However, it was found by [17] that several prominent molecular clouds within $\sim 100 \mathrm{pc}$ from Sgr $\mathrm{A}^{*}$ are hard X-ray sources, their spectra being characteristic of X-ray reflection: their spectra that can be described by a power-law continuum and a prominent $6.4 \mathrm{keV}$ iron emission line, both being attributed to Compton scattering and fluorescence processes.

For these reasons these Molecular Clouds are also referred to as X-ray Reflection Nebulae (XRNe). Established XRNs are the Sgr A, Sgr B and Sgr C complexes (see Fig. 8): the lack of sufficiently bright, persistent irradiating sources nearby led to the hypothesis that the observed XRN emission is the "echo" of an outburst of Sgr A* that happened a few hundred years ago, when its luminosity was $10^{39-40} \mathrm{erg} / \mathrm{s}$.

What we see today would then be the reflected radiation delayed by the light travel time across the $\sim 100$ pc region.

If true, this means that in the recent past our Galactic Center hosted a low-luminosity AGN and we can reconstruct the history of its energy release using the XRNs [5].

We expect this reflected radiation to be strongly polarized [11] at a position angle orthogonal to the direction of the illuminating source and polarization degree related to the source-cloud-observer (scattering) angle.

In Fig. 10 is shown a simulation of the polarization degree and angle measured by IXPE in the Sgr B2 Molecular Cloud in the hypothesis that $\mathrm{Sgr}^{*}$ is the illuminating source.

Aided by its imaging capability in the complex, crowded field of the Galactic Center, IXPE can test unambiguously the hypothesis that $\operatorname{Sgr} \mathrm{A}^{*} \mathrm{X}$-ray luminosity was $10^{6}$ larger $\approx 300$ years ago, making our Galaxy a low-luminosity AGN in the recent past.

If not, it would still be a discovery since alternative hypotheses have been suggested (e.g. [18]).

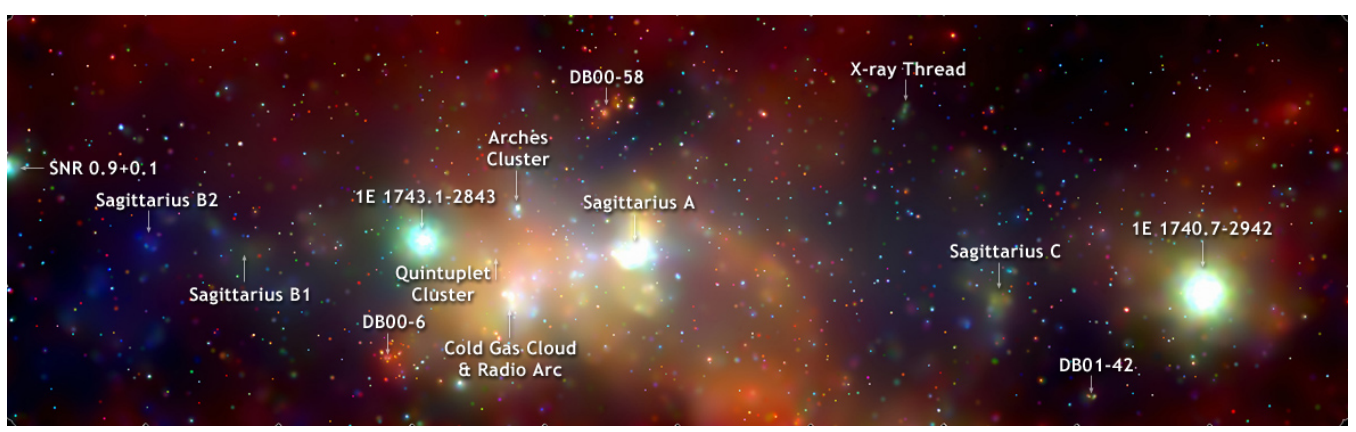

Figure 8: RGB Chandra image of the central 100pc of the Milky Way. In red $1-3 \mathrm{keV}$, in green 3-5 $\mathrm{keV}$, in blue $5-8 \mathrm{keV}$. Labeled are the X-ray reflection nebulae Sgr A, B and C and other prominent X-ray sources. 


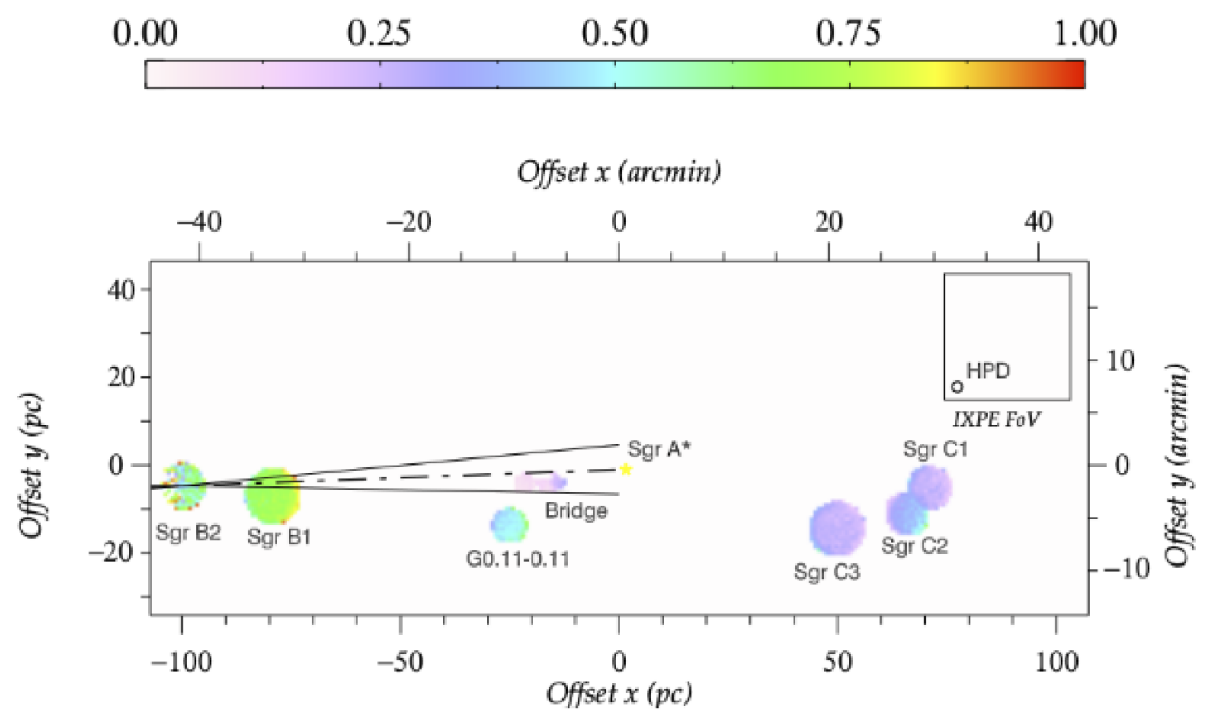

Figure 9: Polarization fraction expected from the molecular clouds around $\operatorname{SgrA}^{*}$ [11].

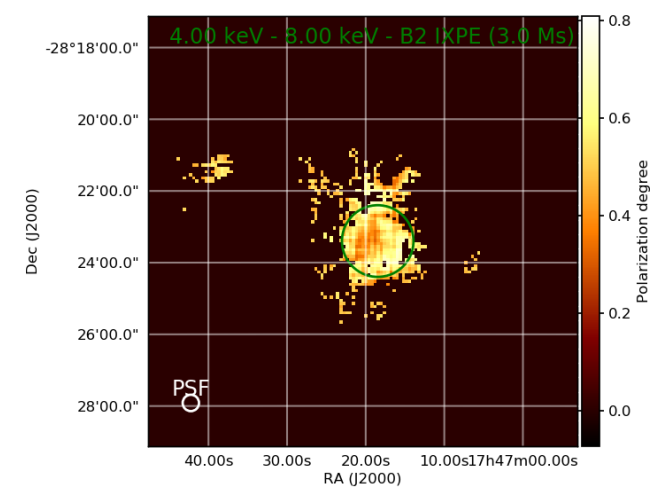

(a)

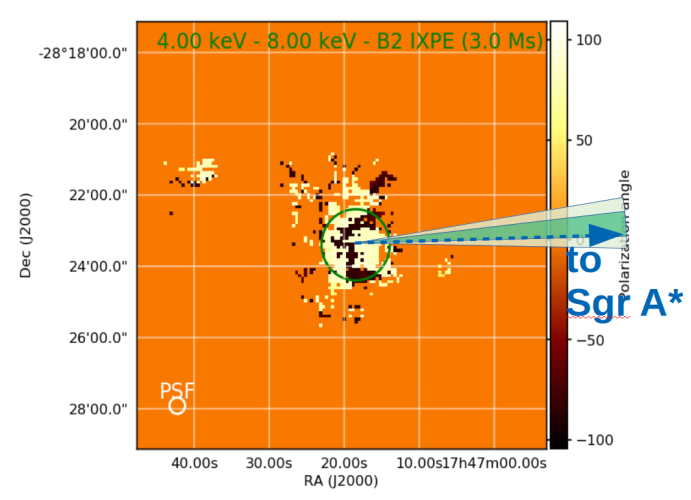

(b)

Figure 10: (a) Polarization degree map. (b) Polarization angle map. In shades of green the reconstructed $1-\sigma$ and $2-\sigma$ direction of the external illuminating source (SgrA* in the employed model).

\section{Conclusions}

IXPE will be the first mission entirely dedicated to the study of X-ray polarimetry and will awake a field that has been dormant for decades.

Thanks to X-ray polarimetry we will be able to probe strong magnetic and gravitational fields, investigate small -scale source geometry trough scattering and identify emission processes.

The imaging capabilities brought by the Gas Pixel Detector will allow to map the magnetic field topology with unprecedented detail and enhance the sensitivity in diffuse and extended sources, bridging the gap with the knowledge already acquired at longer wavelengths. 


\section{Acknowledgments}

I want to thank Fabio Muleri for trusting me to take his place in presenting the IXPE mission at the 2019 Frascati Workshop. I also want to thank Paolo Soffitta, Enrico Costa and Immacolata Donnarumma for the advice and support, and the referee.

IXPE is a NASA Astrophysics Small Explorers (SMEX) mission, managed by MSFC and overseen by the Explorers Program Office at GSFC. The Italian contribution to the IXPE mission is supported by the Italian Space Agency through agreement ASI-INAF n.2017-12-H.0 and ASI-INFN agreement n.2017-13-H.0.

\section{References}

[1] Baganoff, F. K.; Maeda, Y.; Morris, M.; Bautz, M. W.; Brandt, W. N.; Cui, W.; Doty, J. P.; Feigelson, E. D.; Garmire, G. P.; Pravdo, S. H.; Ricker, G. R.; Townsley, L. K., "Chandra X-Ray Spectroscopic Imaging of Sagittarius A* and the Central Parsec of the Galaxy", The Astrophysical Journal, Volume 591, Issue 2, pp. 891-915 (2003).

[2] Bell, A. R., "Turbulent amplification of magnetic field and diffusive shock acceleration of cosmic rays", Monthly Notices of the Royal Astronomical Society, Volume 353, Issue 2, pp. $550-558$ (2004).

[3] Bellazzini, R.; Spandre, G.; Minuti, M.; Baldini, L.; Brez, A.; Latronico, L.; Omodei, N.; Razzano, M.; Massai, M. M.; Pesce-Rollins, M.; Sgró, C.; Costa, Enrico; Soffitta, Paolo; Sipila, H.; Lempinen, E., "A sealed Gas Pixel Detector for X-ray astronomy", Nuclear Instruments and Methods in Physics Research Section A, Volume 579, Issue 2, p. 853-858 (2007).

[4] Bykov, A. M.; Uvarov, Yu. A.; Bloemen, J. B. G. M.; den Herder, J. W.; Kaastra, J. S., " A model of polarized X-ray emission from twinkling synchrotron supernova shells", Monthly Notices of the Royal Astronomical Society, Volume 399, Issue 3, pp. 1119-1125 (2009).

[5] Churazov, E.; Khabibullin, I.; Ponti, G.; Sunyaev, R., "Polarization and long-term variability of Sgr A* X-ray echo", Monthly Notices of the Royal Astronomical Society, Volume 468, Issue 1, p.165-179 (2013).

[6] Costa, E., Soffitta, P., Bellazzini, R., Brez, A., Lumb, N., \& Spandre, G., "An efficient photoelectric X-ray polarimeter for the study of black holes and neutron stars", Nature 411, 662-665 (2001).

[7] Costa, E., "How polarimetry will complete the role of X-rays as e.m. messengers of High Energy Phenomena", 11th Workshop on science with the new generation of High Energy Gamma-ray Experiments (2016).

[8] Donato, D.; Ghisellini, G.; Tagliaferri, G.; Fossati, G., "Hard X-ray properties of blazars", Astronomy and Astrophysics, v.375, p.739-751 (2001).

[9] Fabiani, S., "Instrumentation and Future Missions in the Upcoming Era of X-Ray Polarimetry", Galaxies, 6 (2018).

[10] Malkov, M. A.; Drury, L. O’C., " Nonlinear theory of diffusive acceleration of particles by shock waves", Reports on Progress in Physics, Volume 64, Issue 4, pp. 429-481 (2001).

[11] Marin, F., Muleri, F., Soffitta, P., Karas, V., Kunneriath, D., " Reflection nebulae in the Galactic center: soft X-ray imaging polarimetry ", Astronomy and Astrophysics, 576, A19 (2015).

[12] McNamara, Aimee L.; Kuncic, Zdenka; Wu, Kinwah, "X-ray polarization in relativistic jets", Monthly Notices of the Royal Astronomical Society, Volume 395, Issue 3, pp. 1507-1514 (2009). 
[13] Muleri, F., Lefevre, C., Piazzolla, R., Morbidini, A., Amici, F., Attina, P., Centrone, M., Del Monte, E., Di Cosimo, S., Di Persio, G., Evangelista, Y., Fabiani, S., Ferrazzoli, R., Loffredo, P., Maiolo, L., Maita, F., Primicino, L., Rankin, J., Rubini, A., Santoli, F., Soffitta, P., Tobia, A., Tortosa, A., \& Trois, A., "Calibration of the IXPE instrument," SPIE 10699, 5C 11pp (2018).

[14] Ramsey, B. D., "Optics for the Imaging X-ray Polarimetry Explorer", SPIE 10399, 07 10pp (2017).

[15] Shklovsky, J. S., "Supernovae as a Source of Cosmic Rays", Les Processus Nucléaires dans les Astres, Communications présentées au cinquième Colloque International d'Astrophysique tenu à Liège les 10-12 Septembre, 1953., p.515 (1954).

[16] Soffitta, P., "IXPE the Imaging X-ray Polarimetry Explorer", SPIE 10397, 0I 9pp (2017).

[17] Sunyaev, R. A.; Markevitch, M.; Pavlinsky, M., "The Center of the Galaxy in the Recent Past: A View from GRANAT", Astrophysical Journal v.407, p.606 (1993).

[18] Valinia, A., Tatischeff, V., Arnaud, K., Ebisawa, K., Ramaty, R., "On the Origin of the Iron K Line in the Spectrum of The Galactic X-Ray Background.", The Astrophysical Journal 543, 733 (2000).

[19] Weisskopf, M. C., Cohen, G. G., Kestenbaum, H. L., Long, K. S., Novick, R., \& Wolff, R. S., "Measurement of the X-ray polarization of the Crab Nebula", ApJ 208, L125-L128 (1976).

[20] Weisskopf, M. C., Silver, E. H., Kestenbaum, H. L., Long, K. S., \& Novick, R., "A precision measurement of the X-ray polarization of the Crab Nebula without pulsar contamination", ApJ 220, L117-L121 (1978).

[21] Weisskopf, M.C., Ramsey, B., O’Dell, S.L., Tennant, A., Elsner, R., Soffita, P., Bellazzini, R., Costa, E., Kolodziejczak, J., Kaspi, V., Mulieri, F., Marshall, H., Matt, G., Romani, R., on behalf of the entire IXPE Team, "The Imaging X-ray Polarimetry Explorer (IXPE)", Results in Physics (2016). 


\section{DISCUSSION}

JIM BEALL: You are launching with the orbital ATK launch System. That means that you can have any orbit you want. What is the optimal orbit?

RICCARDO FERRAZZOLI: The optimal orbit is a $540 \mathrm{~km}$ circular orbit at 0 deg inclination: it allows to avoid the worst of the South Atlantic Magnetic Anomaly while guaranteeing a frequent coverage from the Malindi Ground Station (Note: the launcher changed from Pegasus to a Falcon9 rocket, but the answer to the question is still valid).

GENNADY BISNOVATYI-KOGAN: what level of polarization you expect to measure (sensitivity)? Linear or circular?

RICCARDO FERRAZZOLI: We are currently limited by technology to measuring linear X-ray polarization only. As far as sensitivity goes, it will strongly depend from the the brightness of the source and on how long you stare at it. In theory it could be possible to measure as low as a $1 \%$ polarization, but it would be very challenging.

KUMIKO NOBUKAWA: Is the meaning of the observation of the X-ray reflection nebulae just confirmation of the X-ray reflection scenario? Is there any other meaning or something that you expect?

RICCARDO FERRAZZOLI: Yes, I am not aware of other science cases regarding Molecular Clouds: we will use them as "road signs" to point to the (external?) illuminating source. 